\section{(6) OPEN ACCESS}

\title{
Focused HLA analysis in Caucasians with myositis identifies significant associations with autoantibody subgroups
}

Simon Rothwell, ${ }^{\oplus 1}$ Hector Chinoy, ${ }^{\bullet 2,3}$ Janine A Lamb, ${ }_{1}^{4}$ Frederick W Miller, ${ }^{5}$ Lisa G Rider, ${ }^{5}$ Lucy R Wedderburn, ${ }^{6,7}$ Neil J McHugh, ${ }^{\oplus 8}$ Andrew L Mammen, ${ }^{\oplus, 10}$ Zoe E Betteridge, ${ }_{1}^{8}$ Sarah L Tansley, ${ }_{1}^{8,11}$ John Bowes, ${ }_{15} 12$ Jiř́ Vencovský, ${ }^{13}$ Claire T Deakin, ${ }^{6,7}$ Katalin Dankó, ${ }^{14}$ Limaye Vidya, ${ }_{1}^{15}$ Albert Selva-O'Callaghan, ${ }^{16}$ Lauren M Pachman, ${ }^{17}$ Ann M Reed, ${ }^{18}$ Øyvind Molberg, ${ }^{19}$ Olivier Benveniste, ${ }^{20}$ Pernille R Mathiesen, ${ }^{21}$ Timothy R D J Radstake, ${ }_{1}^{22}$ Andrea Doria, ${ }^{23}$ Jan de Bleecker, ${ }^{24}$ Annette T Lee, ${ }^{25}$ Michael G Hanna, ${ }^{26}$ Pedro M Machado, ${ }^{27,28}$ William E Ollier, ${ }^{4,29}$ Peter K Gregersen, ${ }^{30}$ Leonid Padyukov, ${ }^{31}$ Terrance P O'Hanlon, ${ }^{5}$ Robert G Cooper, ${ }^{32}$ Ingrid E Lundberg, ${ }^{31}$ on behalf of Myositis Genetics Consortium (MYOGEN)

Handling editor Josef $S$ Smolen

- Additional material is published online only. To view please visit the journal online (http://dx.doi.org/10.1136/ annrheumdis-2019-215046)

For numbered affiliations see end of article.

Correspondence to Simon Rothwell, Centre for Genetics and Genomics, Arthritis Research UK, University of Manchester, Manchester M13 9PL, UK;

s.rothwell@manchester.ac.uk

Received 9 January 2019 Revised 14 March 2019 Accepted 30 March 2019 Published Online First 28 May 2019

Check for updates

(C) Author(s) (or their employer(s)) 2019. Re-use permitted under CC BY-NC. No commercial re-use. See rights and permissions. Published by BMJ.

To cite: Rothwell $\mathrm{S}$,

Chinoy $\mathrm{H}$, Lamb JA,

et al. Ann Rheum Dis

2019;78:996-1002.

\section{ABSTRACT}

Objectives Idiopathic inflammatory myopathies

(IIM) are a spectrum of rare autoimmune diseases

characterised clinically by muscle weakness and

heterogeneous systemic organ involvement.

The strongest genetic risk is within the major

histocompatibility complex (MHC). Since autoantibody presence defines specific clinical subgroups of IIM, we aimed to correlate serotype and genotype, to identify novel risk variants in the $\mathrm{MHC}$ region that co-occur with IIM autoantibodies.

Methods We collected available autoantibody data in our cohort of 2582 Caucasian patients with IIM. High resolution human leucocyte antigen (HLA) alleles and corresponding amino acid sequences were imputed using SNP2HLA from existing genotyping data and tested for association with 12 autoantibody subgroups.

Results We report associations with eight autoantibodies reaching our study-wide significance level of $p<2.9 \times 10^{-5}$. Associations with the 8.1 ancestral haplotype were found with anti-Jo-1 (HLA-B ${ }^{*} 08: 01$, $p=2.28 \times 10^{-53}$ and HLA-DRB1 ${ }^{*} 03: 01, p=3.25 \times 10^{-9}$ ), anti-PM/SCl (HLA-DQB 1 $\left.{ }^{*} 02: 01, p=1.47 \times 10^{-26}\right)$ and anticN1A autoantibodies (HLA-DRB1*03:01, $p=1.40 \times 10^{-11}$ ). Associations independent of this haplotype were found with anti-Mi-2 (HLA-DRB1 *07:01, $p=4.92 \times 10^{-13}$ ) and anti-HMGCR autoantibodies (HLA-DRB1 *11, $\left.p=5.09 \times 10^{-6}\right)$. Amino acid positions may be more strongly associated than classical HLA associations; for example with anti-Jo-1 autoantibodies and position 74 of HLA-DRB $1\left(p=3.47 \times 10^{-64}\right)$ and position 9 of HLA-B $\left(p=7.03 \times 10^{-11}\right)$. We report novel genetic associations with HLA-DQB1 anti-TIF1 autoantibodies and identify haplotypes that may differ between adult-onset and juvenile-onset patients with these autoantibodies. Conclusions These findings provide new insights regarding the functional consequences of genetic polymorphisms within the MHC. As autoantibodies in IIM correlate with specific clinical features of disease, understanding genetic risk underlying development of autoantibody profiles has implications for future research.

\section{Key messages}

What is already known about this subject?

- The strongest genetic risk among patients with idiopathic inflammatory myopathies (IIM) is thought to be within the human leucocyte antigen (HLA) region in autoantibody defined subgroups.

What does this study add?

- This study shows for the first time genetic differences between adult-onset and juvenileonset patients with anti-TIF1 autoantibodies.

- Amino acid imputation identifies novel associations with autoantibodies that are stronger than classical HLA associations, indicating key positions within HLA molecules that may confer risk.

How might this impact on clinical practice or future developments?

- Differing associations in adult and juvenile onset disease with the same autoantibody suggests distinct aetiologies and disease mechanisms.

- As autoantibodies in myositis correlate with specific clinical features of disease, understanding genetic risk underlying development of certain autoantibody profiles will have implications for future research in IIM.

\section{INTRODUCTION}

The idiopathic inflammatory myopathies (IIM) are a spectrum of rare autoimmune diseases characterised clinically by muscle weakness and systemic organ involvement. Clinically, IIM are heterogeneous and may be subclassified as dermatomyositis (DM), inclusion body myositis (IBM), immune-mediated necrotising myopathy, polymyositis (PM) and antisynthetase syndrome. ${ }^{12}$ 
Myositis autoantibodies can be detected in approximately $60 \%-70 \%$ of children and adults with IIM. ${ }^{34}$ Autoantibodies unique to IIM are known as myositis-specific autoantibodies (MSA), with patients rarely possessing more than one MSA. ${ }^{5}$ Autoantibodies that present in patients with myositis in association with another connective tissue disease are known as myositis-associated autoantibodies (MAA).

Clinical classification of IIM can lead to heterogeneous subgroups, however, some MSA positive subgroups have specific clinical features and may respond differently to treatment. Therefore, autoantibody status may be a more meaningful way to characterise patients to understand pathogenesis and predict prognosis. ${ }^{3}$ For example, patients with anti-histidyl-tRNA-synthetase (anti-Jo-1) commonly present with myositis, Raynaud's phenomenon, polyarthritis and a high frequency of interstitial lung disease. ${ }^{6}$ Patients with autoantibodies directed against antiMi-2 present with hallmark cutaneous manifestations of DM, generally milder myositis and a favourable response to immunosuppressive treatment. ${ }^{7}$

IIM are thought to be complex genetic diseases, initiated by immune activation following specific environmental events in genetically predisposed individuals. The major histocompatibility complex (MHC), also known as the human leucocyte antigen (HLA) region, has consistently been identified as the strongest risk factor for IIM and clinical subgroups. ${ }^{8}$ Studies have shown that the strongest HLA associations among patients with IIM are found when stratifying by autoantibody status. ${ }^{9-11}$ In particular, the strongest risk is with the 8.1 ancestral haplotype (8.1 AH), a common haplotype of extensive linkage disequilibrium (LD) in Caucasian populations that confers susceptibility to IIM and many other autoimmune or immune-mediated diseases. ${ }^{12}$ Associations with alleles independent of the $8.1 \mathrm{AH}$ have also been reported. ${ }^{9}$ New MSAs and MAAs have since been discovered, and small studies have identified potential HLA associations with these autoantibodies, for example, HLA-DRB1*11:01 in patients with anti-3-hydroxy-3-methylglutaryl-coenzyme A reductase (anti-HMGCR) autoantibodies in adult-onset disease. ${ }^{13}$

Here, we aimed to correlate serotype with genotype in patients with IIM, with sufficient statistical power, to identify novel risk variants in the MHC region that confer susceptibility to IIM autoantibodies and to provide a definitive replication cohort for previously reported associations.

\section{METHODS}

\section{Study population}

Through the Myositis Genetics Consortium (MYOGEN), 2582 Caucasian cases from 14 countries were recruited (online supplementary table 1). ${ }^{8}$ Written informed consent was obtained from all patients with approval from research ethics committees of institutional review boards at each participating centre. Patients were enrolled in to MYOGEN if they fulfilled Bohan and Peter criteria for PM and adult and juvenile DM, ${ }^{8}$ or Griggs, Medical Research Council (MRC) or European Neuromuscular Centre (ENMC) criteria for patients with IBM. ${ }^{14}$ Shared control genotypes were drawn from a pool of 15651 individuals from 12 countries as described previously. ${ }^{8}$ Genotypes from an additional 19 UK juvenile-onset patients were included from the Juvenile Dermatomyositis Cohort Biomarker Study and Repository. Patients were classified as having juvenile IIM if age of onset was $<16$ years old, except in the USA, where 18 years old was considered the cut-off.

\section{Autoantibody testing}

Myositis relevant autoantibodies were detected using immunoprecipitation (IP), line blot or ELISA, as detailed in online supplementary table 2. Further details are described in online supplementary method.

\section{Genotyping}

Genotyping was performed in accordance with UK-based and US-based Illumina protocols (Centre for Genetics and Genomics Arthritis Research UK, University of Manchester; Feinstein Institute, New York; University College London, London, UK). Standard quality control (QC) was performed as described previously. ${ }^{8}$

\section{HLA imputation}

Classical HLA alleles and corresponding amino acid sequences were imputed using SNP2HLA with reference data collected by the Type 1 Diabetes Genetics Consortium. ${ }^{15}$ Cases and controls were imputed together. Postimputation QC removed variants with a minor allele frequency $<0.01$ and variants with an information score $<0.8$.

\section{Statistical analysis}

We employed a case-control study design in each autoantibody subgroup analysis. Controls were matched for ethnicity using principal components analysis (PCA), due to absence of geographically matched controls for each IIM cohort. Four healthy controls for each case were matched for ethnicity using (PCA) coordinates, using a method described previously. ${ }^{16}$ Analysis was performed in R (V.3.1.0) on the dosage of variants (genotype probabilities) which takes into account imputation uncertainty. Single-test association was performed for HLA alleles and omnibus tests for multiallelic sites. For multiallelic sites, such as amino acids, we identified the most common residue or allele in the control population to be selected as the reference. A logistic regression assuming an additive model was used to test for association, with gender and the top 10 principal components included as covariates. Forward stepwise logistic regression was used to test for independent effects conditional on the variant of interest. ORs ratios were generated with multivariate logistic regression. Significance was defined as $\mathrm{p}<2.9 \times 10^{-5}$ based on a Bonferroni correction of the pre-QC 1700 variants imputed by SNP2HLA.

\section{RESULTS}

\section{Autoantibody frequencies in the study population}

Genetic data were available for 2582 patients with IIM, the majority of whom were tested for at least one autoantibody. The number of autoantibody positive cases present in the cohort is shown in table 1 , for groups where $\mathrm{n}>10$. Autoantibody frequencies are as a proportion of those in tested individuals and do not necessarily represent the prevalence of autoantibodies in an IIM population.

\section{Strong classical HLA associations are observed in IIM autoantibody subgroups}

Data quality was evaluated after imputation by determining the concordance between imputed alleles and existing HLA typing from next generation sequencing technology (HistoGenetics, New York, USA) for 162 individuals. There was a high level of concordance across all loci for both 2-digit (96.6\%-100\%) and 4-digit (94.8\%-100\%) alleles (online supplementary table 


\section{Myositis}

\begin{tabular}{|c|c|c|c|c|c|}
\hline \multirow[b]{2}{*}{ Antibody } & \multicolumn{2}{|l|}{ Adult-onset IIM } & \multicolumn{2}{|c|}{ Juvenile-onset IIM } & \multirow{2}{*}{$\begin{array}{l}\text { Total number } \\
\text { positive } \\
\text { (Adult+juvenile- } \\
\text { onset) }\end{array}$} \\
\hline & Number tested & Number antibody positive & Number tested & Number antibody positive & \\
\hline Jo-1 & 1937 & $325(16.8 \%)$ & 447 & $7(1.6 \%)$ & 332 \\
\hline TIF1 & 1697 & $91(5.4 \%)$ & 356 & $106(29.8 \%)$ & 197 \\
\hline $\mathrm{PM} / \mathrm{SCl}$ & 1883 & $120(6.4 \%)$ & 457 & $16(3.5 \%)$ & 136 \\
\hline Mi-2 & 1884 & $87(4.6 \%)$ & 456 & $17(3.7 \%)$ & 104 \\
\hline NXP2 & 1502 & $28(1.9 \%)$ & 360 & $65(18.1 \%)$ & 93 \\
\hline CN1A & 232 & $46(19.8 \%)$ & 81 & $18(22.2 \%)$ & 64 \\
\hline SRP & 1822 & $45(2.5 \%)$ & 457 & 0 & 45 \\
\hline HMGCR & 1264 & $51(4.0 \%)$ & 130 & $1(0.8 \%)$ & 52 \\
\hline MDA5 & 1674 & $18(1.1 \%)$ & 402 & $17(4.2 \%)$ & 35 \\
\hline SAE & 1518 & $31(2.0 \%)$ & 397 & 0 & 31 \\
\hline PL7 & 1927 & $23(1.2 \%)$ & 457 & 0 & 23 \\
\hline PL12 & 1927 & $11(0.6 \%)$ & 457 & 0 & 11 \\
\hline
\end{tabular}

Myositis-specific/associated autoantibodies $n>10$ in cohort, stratified by adult-onset and juvenile-onset disease. Frequency is of those patients tested for each autoantibody within the cohort, where testing was not necessarily random.

IIM, idiopathic inflammatory myopathies.

3). After stringent QC removing variants with low frequency $(<0.01)$ and poor imputation accuracy $(\mathrm{r} 2<0.8)$, our analysis included 509 classical HLA alleles and amino acids. In the primary analysis, adult-onset and juvenile-onset cases were combined and analysed together against matched healthy controls. Autoantibody associations reaching study-wide significance of $\mathrm{p}<2.9 \times 10^{-5}$ are reported in table 2 .

The strongest HLA association was found with anti-Jo- 1 autoantibodies and HLA-B*08:01 $\left(\mathrm{p}=2.28 \times 10^{-53}, \mathrm{OR}=3.37,95 \%\right.$ CI 2.37 to 4.83 ). Conditioning on HLA-B*08:01 in a stepwise logistic regression model revealed an independent association with HLA-DRB1*03:01 $\left(\mathrm{p}=3.25 \times 10^{-9}, \mathrm{OR}=3.09,95 \%\right.$ CI 2.08 to 4.30 ). We analysed antisynthetase autoantibody positive patients as a group $(\mathrm{n}=381)$, and the strength of association became stronger (HLA-B $* 08: 01, p=1.79 \times 10^{-56}, \mathrm{OR}=3.14$, $95 \%$ CI 2.27 to 4.38 and HLA-DRB1*03:01, $\mathrm{p}=3.31 \times 10^{-9}$, $\mathrm{OR}=2.73,95 \%$ CI 1.96 to 3.80 ). We did not find associations with rarer aminoacyl-tRNA-synthetases individually, likely due to small sample sizes. However, in a small sample size of 23 patients, only $30.4 \%$ of patients with anti-PL7 antibodies carried HLA-DRB1*03:01, compared with $79.2 \%$ of patients with antiJo- 1 autoantibodies, and $23 \%$ of healthy controls (online supplementary table 4). This may suggest that not all antisynthetase autoantibodies have the same associations with $8.1 \mathrm{AH}$. The 'haplotype phased' raw HLA genotypes for patients with rarer antisynthetase autoantibodies are included in online supplementary tables 5-10.

A strong HLA association was observed in patients with anti-TIF1 autoantibodies with the HLA-allele group DQB1*02 $\left(\mathrm{p}=2.34 \times 10^{-10}, \quad \mathrm{OR}=2.49,95 \%\right.$ CI 1.88 to 3.31). Within this allele group, there were associations with HLA-DQB1*02:01 $\left(\mathrm{p}=1.69 \times 10^{-5}\right)$ and HLA-DQB1*02:02 $\left(\mathrm{p}=1.04 \times 10^{-4}\right)$.

Associations with class II alleles of the $8.1 \mathrm{AH}$ were observed for patients with anti-Jo-1, anti-PM/Scl and anti-cN1A. AntiHMGCR and anti-Mi-2 were the only autoantibodies tested that were associated with HLA alleles not forming part of the 8.1 $\mathrm{AH}$. Although the strong association with anti-HMGCR autoantibodies and HLA-DRB1*11 was confirmed in adult-onset patients, a stronger association was observed with the 4-digit allele HLA-DRB1*11:01 ( $\mathrm{p}=3.22 \times 10^{-7}, \mathrm{OR}=11.90,95 \% \mathrm{CI}$ 4.72 to 31.85 ) before the allele was removed from the initial analysis due to low imputation accuracy $\left(r^{2}=0.75\right)$.

Using a more conservative threshold correcting for the number of antibodies analysed $\left(\mathrm{p}<2.4 \times 10^{-6}\right)$, the association with HLA-DRB*11 and anti-HMGCR autoantibodies did not reach our significance threshold. There were no significant associations with classical HLA alleles and anti-NXP2, anti-SRP, anti-MDA5 or anti-SAE autoantibodies.

Table 2 Classical HLA allele associations

\begin{tabular}{llllll}
\hline \multirow{2}{*}{ Autoantibody } & & \multicolumn{5}{l}{ SNP2HLA classical HLA association } & & \\
\cline { 2 - 6 } & Number of cases & Gene & Allele & P value & OR (95\% CI) \\
\hline Jo-1 & 332 & HLA-B & $08: 01$ & $2.28 \times 10^{-53}$ & $3.37(2.37$ to 4.83$)$ \\
& & HLA-DRB1 & $03: 01$ & $3.25 \times 10^{-9}$ & $3.09(2.08$ to 4.30$)$ \\
TIF1 & 197 & HLA-DQB1 & 02 & $2.34 \times 10^{-10}$ & $2.49(1.88$ to 3.31$)$ \\
PM/SCl & 136 & HLA-DQB1 & $02: 01$ & $1.47 \times 10^{-26}$ & $17.50(10.63$ to 30.59$)$ \\
Mi-2 & 104 & HLA-DRB1 & $07: 01$ & $4.92 \times 10^{-13}$ & $5.47(3.48$ to 8.77$)$ \\
CN1A & 64 & HLA-DRB1 & $03: 01$ & $1.40 \times 10^{-11}$ & $9.23(4.95$ to 18.01$)$ \\
HMGCR & 52 & HLA-DRB1 & 11 & $5.09 \times 10^{-6}$ & $4.92(2.52$ to 9.97$)$ \\
\hline
\end{tabular}

4-digit alleles are preferentially reported unless a 2-digit allele is significantly more associated. P values (uncorrected), OR and $95 \% \mathrm{Cl}$ were calculated in a regression including the most significantly associated independent variants. Associations reaching study-wide significance are reported $\left(\mathrm{p}<2.9 \times 10^{-5}\right)$. HLA, human leucocyte antigen. 
Table 3 The strongest amino acid associations in autoantibody subgroups of IIM

\begin{tabular}{|c|c|c|c|c|c|}
\hline \multirow[b]{2}{*}{ Autoantibody } & \multirow[b]{2}{*}{ Number of cases } & \multicolumn{4}{|c|}{ Amino acid association } \\
\hline & & Gene & Variant & $P$ value & OR $(95 \% \mathrm{Cl})$ \\
\hline \multirow[t]{10}{*}{ Jo-1 } & 332 & HLA-DRB1 & Position 74 & Omnibus $3.47 \times 10^{-64}$ & \\
\hline & & & Alanine (ref) & & \\
\hline & & & Arginine & $1.24 \times 10^{-8}$ & 2.94 (2.03 to 4.26 ) \\
\hline & & & Glutamine & 0.02 & 0.59 (0.38 to 0.89$)$ \\
\hline & & & Leucine & $6.21 \times 10^{-5}$ & 2.78 (1.67 to 4.55$)$ \\
\hline & & & Glutamic acid & 0.5 & 1.20 (0.70 to 1.96$)$ \\
\hline & & HLA-B & Position 9 & Omnibus $7.03 \times 10^{-11}$ & \\
\hline & & & Tyrosine (ref) & & \\
\hline & & & Aspartic acid & $2.10 \times 10^{-10}$ & 3.33 (2.30 to 4.84$)$ \\
\hline & & & Histidine & 0.77 & 0.96 (0.73 to 1.26$)$ \\
\hline \multirow[t]{3}{*}{ TIF1 } & 197 & HLA-DQB1 & Position 37 & Omnibus $1.49 \times 10^{-10}$ & \\
\hline & & & Tyrosine (ref) & & \\
\hline & & & Isoleucine & $2.34 \times 10^{-10}$ & 2.49 (1.88 to 3.31$)$ \\
\hline \multirow[t]{6}{*}{$\mathrm{PM} / \mathrm{Scl}$} & 136 & HLA-DRB1 & Position 74 & Omnibus $2.48 \times 10^{-40}$ & \\
\hline & & & Alanine (ref) & & \\
\hline & & & Arginine & $5.06 \times 10^{-25}$ & 18.37 (10.88 to 32.98$)$ \\
\hline & & & Glutamine & 0.43 & 1.26 (0.70 to 2.21$)$ \\
\hline & & & Leucine & 0.58 & 0.70 (0.17 to 2.32$)$ \\
\hline & & & Glutamic acid & 0.58 & 1.34 (0.44 to 3.56$)$ \\
\hline \multirow[t]{3}{*}{ Mi-2 } & 104 & HLA-DRB1 & Position 4 & Omnibus $3.69 \times 10^{-13}$ & \\
\hline & & & Arginine (ref) & & \\
\hline & & & Glutamine & $3.26 \times 10^{-12}$ & 4.93 (3.17 to 7.79$)$ \\
\hline \multirow[t]{6}{*}{$\mathrm{cN1A}$} & 64 & HLA-DRB1 & Position 74 & Omnibus $6.15 \times 10^{-14}$ & \\
\hline & & & Alanine (ref) & & \\
\hline & & & Arginine & $1.37 \times 10^{-9}$ & 8.68 (4.43 to 18.1$)$ \\
\hline & & & Glutamine & 0.02 & 0.17 (0.03 to 0.61$)$ \\
\hline & & & Leucine & 0.05 & 3.31 (0.93 to 11.02$)$ \\
\hline & & & Glutamic acid & 0.43 & 0.45 (0.03 to 2.30$)$ \\
\hline \multirow[t]{6}{*}{ SRP } & 45 & HLA-DRB1 & Position 74 & Omnibus $1.91 \times 10^{-5}$ & \\
\hline & & & Alanine (ref) & & \\
\hline & & & Arginine & $9.47 \times 10^{-4}$ & 3.51 (1.69 to 7.57 ) \\
\hline & & & Glutamine & 0.13 & 0.38 (0.08 to 1.18$)$ \\
\hline & & & Leucine & 0.63 & 1.36 (0.32 to 4.34$)$ \\
\hline & & & Glutamic Acid & $5.69 \times 10^{-4}$ & 5.07 (2.05 to 13.17$)$ \\
\hline \multirow[t]{3}{*}{ HMGCR } & 42 & HLA-DRB1 & Position 58 & Omnibus $2.42 \times 10^{-6}$ & \\
\hline & & & Alanine (ref) & & \\
\hline & & & Glutamic acid & $5.13 \times 10^{-6}$ & 4.91 (2.52 to 9.97$)$ \\
\hline \multirow[t]{5}{*}{ SAE } & 31 & HLA-DQB1 & Position 57 & Omnibus $2.66 \times 10^{-6}$ & \\
\hline & & & Aspartic acid (ref) & & \\
\hline & & & Alanine & $1.71 \times 10^{-5}$ & 8.52 (3.42 to 24.47$)$ \\
\hline & & & Valine & 0.41 & 1.61 (0.49 to 4.92$)$ \\
\hline & & & Serine & 0.94 & 0.92 (0.04 to 7.66$)$ \\
\hline
\end{tabular}

OR and $95 \% \mathrm{Cl}$ were calculated in a regression model including significant independent variants, using the most common amino acid in the population as the reference. Associations reaching study-wide significance are reported $\left(p<2.9 \times 10^{-5}\right)$.

IIM, idiopathic inflammatory myopathies.

\section{Amino acid positions may be more strongly associated than classical HLA alleles}

SNP2HLA was used to impute amino acid positions. We employed a case-control study design in each autoantibody subgroup analysis, and autoantibodies with significant amino acid associations are reported in table 3 .

The omnibus $\mathrm{p}$ value reports the significance of the amino acid position, and the effect sizes of the potential amino acids at this location were calculated in a regression model. The location of associated amino acids within the 3D structure of HLA molecules are shown in online supplementary figure 1 . For some autoantibodies, such as anti-Jo-1, anti-PM/Scl and anti-cN1A, amino acid associations were markedly stronger than the classical HLA associations reported in table 2. Regional association plots for autoantibodies with significant associations are shown in online supplementary figure $2 \mathrm{~A}-\mathrm{H}$. Using a more conservative threshold correcting for the number of antibodies analysed $\left(\mathrm{p}<2.4 \times 10^{-6}\right)$, associations with amino acids and anti-SRP, antiHMGCR and anti-SAE autoantibodies did not reach our significance threshold.

For anti-Jo-1, anti-PM/Scl, anti-cN1A and anti-SRP autoantibodies, the strongest association was with position 74 of 
Table 4 Differences in association at the HLA-DQB1 *02 locus in adult-onset and juvenile-onset patients with anti-TIF1 autoantibodies

\begin{tabular}{lll}
\hline & $\mathrm{DQB1}$ *02:01 & $\mathrm{DQB1}$ *02:02 \\
\hline Adult-onset TIF1, $\mathrm{n}=91$ & $\mathrm{P}=0.06$ & $\mathrm{P}=2.96 \times 10^{-5}$ \\
& $\mathrm{OR}=1.54(0.97$ to 2.44$)$ & $\mathrm{OR}=3.31(1.89-5.84)$ \\
Juvenile-onset TIF1, $\mathrm{n}=106$ & $\mathrm{P}=3.70 \times 10^{-5}$ & $\mathrm{P}=0.13$ \\
& $\mathrm{OR}=2.47(1.61$ to 3.80$)$ & $\mathrm{OR}=1.50$ (0.87 to 2.51$)$ \\
\hline
\end{tabular}

OR with $95 \% \mathrm{Cl}$.

HLA-DRB1 (table 3). For anti-Jo-1, anti-PM/Scl and anticN1A autoantibodies, an arginine at position 74 of HLA-DRB1 conferred the strongest risk. In patients with anti-PM/Scl and anti-cN1A autoantibodies, an arginine conferred all of the risk at this position, whereas in patients with anti-Jo-1 autoantibodies there was also evidence of risk attributable to other amino acids.

Stepwise conditional analysis showed evidence of multiple independent effects in the HLA region with anti-Jo-1; position 74 of HLA-DRB1 and position 9 of HLA-B. These were associated at a similar level of significance $\left(p=3.47 \times 10^{-64}\right.$ vs $p=5.25 \times 10^{-64}$ ) and remained significant after conditioning on either position. Analysing antisynthetase autoantibody positive patients as a group increased the strength of association with these independent amino acid positions (HLA-DRB1 Position $74, p=5.69 \times 10^{-67}$ and HLA-B Position 9, $\left.p=8.63 \times 10^{-11}\right)$.

\section{HLA alleles may differentiate adult-onset and juvenile-onset patients with anti-TIF1 autoantibodies}

For autoantibodies that occur at high frequencies in both adultonset and juvenile-onset patients with IIM, the cohort was stratified to investigate potential differences in HLA associations between these populations. Patients with anti-TIF1 autoantibodies were stratified into adult-onset $(\mathrm{n}=91)$ and juvenile-onset $(n=106)$ disease. Of these, all but six adult-onset patients were recorded as having DM. The association at the HLA-DQB1 locus differed between adult-onset and juvenile-onset patients (table 4). In adult-onset IIM, the strongest 4-digit HLA association was with HLA-DQB1*02:02 $\left(\mathrm{p}=2.96 \times 10^{-5}, \mathrm{OR}=3.31\right.$, $95 \%$ CI 1.89 to 5.84). In contrast, in juvenile-onset patients, a strong association with HLA-DQB1*02:01 was observed, but the strongest association was with HLA-DRB1*03:01 $\left(\mathrm{p}=6.21 \times 10^{-}\right.$ ${ }^{6}, \mathrm{OR}=2.69,95 \% \mathrm{CI} 1.75$ to 4.15 ), which is on the same haplotype as HLA-DQB1*02:01.

To ensure that this difference was not due to the effects of different methods of autoantibody detection, analysis was restricted to UK patients serotyped using IP in the same centre at the University of Bath. Nineteen additional juvenile-onset patients were included, recently genotyped through the Juvenile Dermatomyositis Cohort Biomarker Study and Repository. This subanalysis included UK adult-onset $(n=40)$ and juvenile-onset patients $(n=48)$ with anti-TIF1 autoantibodies. The strongest associations for juvenile-onset patients were with alleles of the 8.1 $\mathrm{AH}$, which were not significant in the adult-onset cohort. For example, HLA-DQB1*02:01 had a stronger association in the juvenile-onset cohort $(\mathrm{p}=0.004, \mathrm{OR}=2.48,95 \%$ CI 1.33 to 4.61) in comparison to the adult-onset cohort $(\mathrm{p}=0.11$, $\mathrm{OR}=1.69,95 \%$ CI 0.87 to 3.20 ). Conversely, HLA-DQB1*02:02 was more strongly associated in adult-onset $(\mathrm{p}=0.01, \mathrm{OR}=2.38$, 95\% CI 1.16 to 4.76$)$ than in juvenile-onset disease ( $\mathrm{p}=0.09$, $\mathrm{OR}=1.85,95 \% \mathrm{CI} 0.89$ to 3.64 ), thus replicating trends from the original cohort. However, due to the reduced sample size, these associations did not reach study-wide significance. These trends were also seen when restricting the analysis to US juvenile-onset patients with anti-TIF1 autoantibodies $(n=70)$; the association with HLA-DQB1*02:01 $(\mathrm{p}=0.01, \mathrm{OR}=2.05,95 \%$ CI 1.18 to 3.54 ) was stronger than with HLA-DQB1*02:02 $(\mathrm{p}=0.76, \mathrm{OR}=0.89,95 \% \mathrm{CI} 0.40$ to 1.81$)$.

There were no significant classical HLA or amino acid associations in either adult-onset or juvenile-onset patients with anti-NXP2 or anti-MDA5 autoantibodies. For anti-Jo-1, anti-PM/Scl, anti-Mi-2 and anti-cN1A, the numbers in the juvenile-onset cohorts were too small to reach our study-wide significance. However, for alleles associated in the total cohort, the trends for association were in the same direction in juvenile-onset IIM (online supplementary table 11).

\section{DISCUSSION}

We used HLA imputation to find associations with IIM autoantibodies and both classical HLA alleles and amino acid positions that may confer risk. By applying this novel approach, we report for the first genetic differences between adult-onset and juvenile-onset anti-TIF1-positive patients. There are independent associations of HLA-DRB1 and HLA-B for the development of anti-Jo-1 autoantibodies, implicating multiple genetic features of the $8.1 \mathrm{AH}$. Amino acid imputation identified novel associations with autoantibodies that are stronger than the classical HLA allelic association, indicating key positions within HLA molecules that may confer risk for various antibodies.

For certain autoantibodies, the strongest amino acid associations were at a similar level of significance as the strongest specific HLA association (online supplementary figure 2A-H). These amino acids may act as a proxy for the risk allele due to carriage on risk haplotypes. In other instances, amino acid associations were markedly more significant than any HLA allele. For example, in patients with anti-PM/Scl autoantibodies, amino acid position 74 of HLA-DRB1 had a stronger association $\left(\mathrm{p}=2.48 \times 10^{-40}\right)$ than any allelic association $\left(\mathrm{p}=1.47 \times 10^{-26}\right)$. For patients with anti-Jo-1 autoantibodies, forward stepwise conditional analysis suggested independent associations within the 8.1 AH of HLA-B*08:01 and HLA-DRB1*03:01. Other MSAs may have multiple independent associations within the HLA region that we were underpowered to detect, in line with other seropositive autoimmune diseases. ${ }^{17}$

Imputation of classical HLA alleles confirmed strong associations between alleles of the $8.1 \mathrm{AH}$ and anti-Jo-1, anti-PM/Scl and anti-cN1A autoantibodies. Specific HLA alleles are thought to target particular autoantigens, resulting in a breakdown in immunological tolerance to self-antigens. However, studies have shown that there are additional genetic features of the $8.1 \mathrm{AH}$ that predispose individuals to immune-mediated diseases, such as NF-kappaB and TNF-alpha polymorphisms, ${ }^{18} 19$ and gene copy number variants of complement genes. ${ }^{20}$ The association of alleles independent of the $8.1 \mathrm{AH}$ with anti-Mi-2 and anti-HMGCR, as well as finding that some autoantibodies do not have strong HLA associations, suggests that not all patients with IIM share a common genetic risk. The 8.1 AH may be more critical for generation of a particular autoantibody repertoire rather than for the development of IIM. Environmental triggers including viruses, infections, UV radiation, drugs and additional genetic risk factors may contribute to disease heterogeneity.

The position of amino acids may give insight into whether these associations are functionally important. For example, amino acid position 74 faces inwards of the peptide binding groove in HLA DR molecules and may change the structure to accommodate autoantigenic peptides, whereas amino acid position 4 lies outside of the peptide binding groove (online supplementary figure 1). 
An arginine at position 74 of HLA-DRB1 was the most strongly associated amino acid position in patients with anti-Jo-1, anti-PM/ $\mathrm{Scl}$ and anti-cN1A autoantibodies and has been implicated in other autoimmune diseases in Caucasian individuals. ${ }^{21}$ Arg-74 almost exclusively resides on HLA-DRB1*03 alleles, which may explain the strong association with many IIM autoantibodies and HLA-DRB1*03:01. It will be interesting to examine whether risk alleles in other ethnic populations share the same amino acids as in Caucasian populations. ${ }^{22}$ Our method of analysis was unable to distinguish whether risk was attributable to amino acid motifs or to individual amino acid positions due to the high level of $\mathrm{LD}$ within these molecules. Previous studies have identified HLA-DRB1 motif ${ }^{9}$ EYSTS $^{13}$ as risk for IIM, ${ }^{23}$ and the HLA-DRB1 motif ${ }^{70} \mathrm{QKXXR}^{74}$ for patients with anti-Jo-1 and anti-PM/Scl autoantibodies. ${ }^{9}$ In both of these cases, however, the motifs are no stronger than the classical association with HLA-DRB1*03:01, or indeed an arginine at position 74 of HLA-DRB1.

We found evidence of different haplotypic associations for adult-onset and juvenile-onset patients with anti-TIF1 autoantibodies. After stratifying by age, there was an association between anti-TIF1 autoantibodies and the $8.1 \mathrm{AH}$ in juvenile-onset patients that was absent in adult-onset patients. Differences in association could be due to distinct aetiologies or to epitope spreading. An association of anti-TIF1 autoantibodies with cancer in adult-onset disease is not present in juvenile-onset disease. ${ }^{24} 25$ Cancer-associated myositis may develop as a paraneoplastic process, with neoantigens in the cancer triggering autoimmunity in adult disease. ${ }^{26}$ In juvenile-onset patients with anti-TIF1 autoantibodies, however, an association with the $8.1 \mathrm{AH}$ may suggest a different aetiology, where environmental risk factors such as infections, ${ }^{27}{ }^{28} \mathrm{UV}$ exposure $^{29}$ or others, ${ }^{30}$ may initiate disease. Differences in association with anti-HMGCR autoantibodies in adult-onset patients (HLADRB1*11:01) and juvenile onset-patients (HLA*DRB1*07:01) have recently been described. ${ }^{13}$

There are some limitations with our study. Due to the nature of this large multicentre collection, not all patients were tested using gold standard IP. Alternatively, patients were included if they tested positive for an autoantibody by a commercially available line blot. Although concordance between these different methods has not been comprehensively studied, there is evidence that these assays are robust. ${ }^{31}{ }^{32} \mathrm{~A}$ subanalysis of patients with anti-Jo- 1 and anti-PM/Scl autoantibodies serotyped using EUROIMMUN line blots resulted in the same associations as those serotyped by IP (data not shown). Another limitation is the potential mischaracterisation of patients as antibody negative. Patients may have rare autoantibodies not currently included on commercially available line-blots, undiscovered antibodies not detectable in cell lines used for IP or low/negative titres of antibody during disease remission. Mischaracterising patients as antibody negative would result in a type-2 error and should not affect our primary findings. For these reasons, we did not compare antibody positive against antibody negative-patient groups.

In conclusion, these results confirm strong associations between HLA alleles and autoantibody specificities present in Caucasian patients with IIM. As well as strong associations with the $8.1 \mathrm{AH}$, we show that there are associations independent of this haplotype and that risk within this region may differ between adult-onset and juvenile-onset patients with anti-TIF1 autoantibodies. As autoantibodies in myositis correlate with specific clinical features of disease, understanding the mechanisms behind development of different autoantibodies should be a focus of ongoing research.

\section{Author affiliations}

${ }^{1}$ Centre for Genetics and Genomics, Arthritis Research UK, University of Manchester, Manchester, UK

${ }^{2}$ National Institute for Health Research Manchester Biomedical Research Centre, Central Manchester University Hospitals NHS Foundation Trust, Manchester, UK ${ }^{3}$ Manchester Academic Health Science Centre, Salford Royal NHS Foundation Trust, Salford, UK

${ }^{4}$ Centre for Epidemiology, The University of Manchester, Manchester, UK

${ }^{5}$ Environmental Autoimmunity Group, National Institute of Environmental Health Sciences, Bethesda, Maryland, USA

${ }^{6} \mathrm{NIHR}$ Great Ormond Street Biomedical Research Centre, University College London, London, UK

${ }^{7}$ Arthritis Research UK Centre for Adolescent Rheumatology, University College London, London, UK

${ }^{8}$ Pharmacy and Pharmacology, University of Bath, Bath, UK

${ }^{9}$ Muscle Disease Unit, Laboratory of Muscle Stem Cells and Gene Regulation,

National Institute of Musculoskeletal and Skin Diseases, National Institutes of Health,

Bethesda, Maryland, USA

${ }^{10}$ Departments of Neurology and Medicine, Johns Hopkins University School of

Medicine, Baltimore, Maryland, USA

${ }^{11}$ Royal National Hospital for Rheumatic Diseases, Royal United Hospitals Bath NHS

Foundation Trust, Bath, UK

${ }^{12}$ Arthritis Research UK Centre for Genetics and Genomics, The University of

Manchester, Manchester, UK

${ }^{13}$ Institute of Rheumatology and Department of Rheumatology, Charles University,

Prague, Czech Republic

${ }^{14}$ Internal Medicine, University of Debrecen, Debrecen, Hungary

${ }^{15}$ Rheumatology Unit, Royal Adelaide Hospital, University of Adelaide, Adelaide,

South Australia, Australia

${ }^{16}$ Internal Medicine Department, Vall d'Hebron General Hospital, Universitat

Autonoma de Barcelona, Barcelona, Spain

${ }^{17}$ Ann \& Robert H. Lurie Children's Hospital of Chicago, Northwestern University,

Feinberg School of Medicine, Chicago, Illinois, USA

${ }^{18}$ Pediatrics, Duke University, Durham, North Carolina, USA

${ }^{19}$ Department of Rheumatology, University of Oslo, Oslo, Norway

${ }^{20}$ Department of Internal Medicine and Clinical Immunology, Pitié-Salpêtrière University Hospital, France, France

${ }^{21}$ Department of Paediatrics and Adolescent Medicine, Rigshospitalet, Copenhagen, Denmark

${ }^{22}$ Department of Rheumatology and Clinical Immunology, Utrecht Medical Center, Utrecht The Netherlands

${ }^{23}$ Division of Rheumatology, University of Padova, Padova, Italy

${ }^{24}$ Department of Neurology, Ghent University, Ghent, Belgium

${ }^{25}$ Robert S. Boas Center for Genomics and Human Genetics, The Feinstein Institute for Medical Research, Manhasset, New York, USA

${ }^{26}$ MRC Centre for Neuromuscular Diseases, Department of Neuromuscular Diseases, University College London Institute of Neurology, London, UK

${ }^{27}$ Department of Rheumatology, University College London Hospital NHS Foundation Trust, London, UK

${ }^{28}$ Department of Rheumatology, Northwick Park Hospital, London North West University Healthcare NHS Trust, London, UK

${ }^{29}$ School of Healthcare Sciences, Manchester Metropolitan University, Manchester, UK

${ }^{30} \mathrm{Center}$ for Genomics and Human Genetics, The Feinstein Institute for Medical

Research, Manhasset, New York, USA

${ }^{31}$ Division of Rheumatology, Department of Medicine, Karolinska University Hospital, Stockholm, Sweden

${ }^{32}$ MRC/ARUK Centre for Integrated Research into Musculoskeletal Ageing, University of Liverpool, Liverpool, , UK

Acknowledgements We thank Mr. Paul New (Salford Royal Foundation Trust) for ethical and technical support. We thank all of the patients and their families who contributed to this study. Thanks to Douglas Bell (NIH/NIEHS) and Elaine Remmers (NIH/NHGRI) for critical review of the manuscript. The authors would like to acknowledge the assistance given by IT Services at The University of Manchester. A full list of acknowledgments is included in the supplementary materials.

Collaborators The following members of the US Childhood Myositis Heterogeneity Study Group contributed to this study: Drs Barbara S Adams (University of Michigan, Ann Arbor, MI), Catherine A Bingham (Hershey Medical Center, Hershey, PA), Gail D Cawkwell (All Children's Hospital, St. Petersburg, FL), Terri H Finkel (Children's Hospital of Philadelphia, Philadelphia, PA), Steven W George (Ellicott City, MD), Harry L Gewanter (Richmond, VA), Ellen A. Goldmuntz (Children's National Medical Center, Washington, DC), Donald P Goldsmith (St Christopher's Hospital for Children, Philadelphia, PA), Michael Henrickson (Children's Hospital, Madera, CA), Lisa Imundo (Columbia University, New York, NY), Ildy M Katona (Uniformed Services University, Bethesda, MD), Carol B Lindsley (University of Kansas, Kansas City), Chester P Oddis (University of Pittsburgh, Pittsburgh, PA), Judyann C Olson (Medical College of Wisconsin, Milwaukee), David Sherry (Children's Hospital of 
Philadelphia, Philadelphia, PA), Scott A Vogelgesang (Walter Reed Army Medical Center, Washington, DC), Carol A Wallace (Children's Medical Center, Seattle, WA), Patience H White (George Washington University, Washington, DC) and Lawrence S Zemel (Connecticut Children's Hospital, Hartford).

Contributors JAL, RGC, IEL, FWM, LGR, NJMCH and HC devised the study concept and design and obtained funding. SR, HC and JAL wrote the manuscript. SR performed the statistical analysis. JB contributed statistical support. JAL, RGC, IEL, FWM, NJMCH, HC, LGR, LP and LRW contributed to the interpretations of findings. Other authors contributed samples and/or data and all authors contributed to and approved the manuscript.

Funding This study was supported in part by: Association Francaise Contre Les Myopathies (AFM), The European Union Sixth Framework Programme (project AutoCure; LSH-018661), European Science Foundation (ESF) in the framework of the Research Networking Programme European Myositis Network (EUMYONET), The Swedish Research Council and grants provided by the Stockholm County Council (ALF project), the Swedish Rheumatism Association, King Gustaf V 80 year Foundation, the intramural research programs of the National Institute of Environmental Health Sciences (NIEHS) and the National Institute of Arthritis and Musculoskeletal and Skin Diseases (NIAMS), the National Institutes of Health (NIH), European Community's FP6, AutoCure LSHB CT-2006-018661, The UK Myositis Support Group, Arthritis Research UK (18474 and 20380), Medical Research Council (MR/N003322/1), The Cure JM Foundation, the Wellcome Trust, the Henry Smith Charity UK, Action Medical UK. The Czech cohort was supported by Project for Conceptual Development of Research Organization 00023728 from Ministry of Health in the Czech Republic. Antibody testing in the UK JDRG cohort was supported by a BMA Dorris Hillier Grant 2012 and the Bath Institute of Rheumatic Diseases. Thank you to the Research Fund of Region Zeeland, Denmark. Pedro M. Machado is supported by the National Institute for Health Research (NIHR) University College London Hospitals (UCL) Biomedical Research Centre (BRC). LRW is supported by the National Institute for Health Research (NIHR) Great Ormond Street Hospital (GOSH) Biomedical Research Centre (BRC). This report includes independent research supported by the NIHR Biomedical Research Centre Funding Scheme.

Disclaimer The views expressed in this publication are those of the authors and not necessarily those of the NHS, the National Institute for Health Research or the Department of Health.

Competing interests JV reports grants from Ministry of Health in the Czech Republic, grants from European Community's FP6, AutoCure LSHB CT-2006-01866, grants from European Science Foundation, during the conduct of the study. IEL reports grants from Swedish Research Council, grants from European Science Foundation, grants from Association Francaise Contre Les Myopathies (AFM), grants from Stockholm County Council, grants from The European Union Sixth Framework Programme, during the conduct of the study.

Patient consent for publication Not required.

Ethics approval Written informed consent was obtained from all cases with approval from research ethics boards at each participating centre.

Provenance and peer review Not commissioned; externally peer reviewed.

Data sharing statement No data are available.

Open access This is an open access article distributed in accordance with the Creative Commons Attribution Non Commercial (CC BY-NC 4.0) license, which permits others to distribute, remix, adapt, build upon this work non-commercially, and license their derivative works on different terms, provided the original work is properly cited, appropriate credit is given, any changes made indicated, and the use is non-commercial. See: http://creativecommons.org/licenses/by-nc/4.0/.

\section{REFERENCES}

1 Lundberg IE, Tjärnlund A, Bottai M, et al. European League against Rheumatism/ American College of rheumatology classification criteria for adult and juvenile idiopathic inflammatory myopathies and their major subgroups. Arthritis Rheumatol 2017:2017:2271-82

2 Mariampillai K, Granger B, Amelin D, et al. Development of a new classification system for idiopathic inflammatory myopathies based on clinical manifestations and myositis-specific autoantibodies. JAMA Neurol 2018;75.

3 Betteridge Z, McHugh N. Myositis-specific autoantibodies: an important tool to support diagnosis of myositis. J Intern Med 2016;280:8-23.

4 Tansley SL, Simou S, Shaddick G, et al. Autoantibodies in juvenile-onset myositis: their diagnostic value and associated clinical phenotype in a large UK cohort. J Autoimmun 2017;84:55-64.

5 McHugh NJ, Tansley SL, myositis Ain. Autoantibodies in myositis. Nat Rev Rheumatol 2018;14:290-302.

6 Lega J-C, Fabien N, Reynaud Q, et al. The clinical phenotype associated with myositisspecific and associated autoantibodies: a meta-analysis revisiting the so-called antisynthetase syndrome. Autoimmun Rev 2014;13:883-91.
7 Love LA, Leff RL, Fraser DD, et al. A new approach to the classification of idiopathic inflammatory myopathy: myositis-specific autoantibodies define useful homogeneous patient groups. Medicine 1991;70:360-74.

8 Rothwell S, Cooper RG, Lundberg IE, et al. Dense genotyping of immune-related loci in idiopathic inflammatory myopathies confirms HLA alleles as the strongest genetic risk factor and suggests different genetic background for major clinical subgroups. Ann Rheum Dis 2016;75:1558-66.

9 O'Hanlon TP, Carrick DM, Targoff IN, et al. Immunogenetic risk and protective factors for the idiopathic inflammatory myopathies: distinct HLA-A, -B, -CW, -DRB1, and -DQA1 allelic profiles distinguish European American patients with different myositis autoantibodies. Medicine 2006:85:111-27.

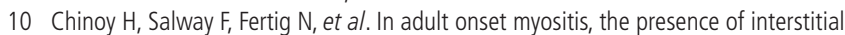
lung disease and myositis specific/associated antibodies are governed by HLA class II haplotype, rather than by myositis subtype. Arthritis Res Ther 2006;8.

11 Miller FW, Chen W, O'Hanlon TP, et al. Genome-wide association study identifies HLA 8.1 ancestral haplotype alleles as major genetic risk factors for myositis phenotypes. Genes Immun 2015;16:470-80.

12 Price $P$, Witt $C$, Allcock $R$, et al. The genetic basis for the association of the 8.1 ancestral haplotype (A1, B8, DR3) with multiple immunopathological diseases. Immunol Rev 1999;167:257-74.

13 Mammen AL, Gaudet D, Brisson D, et al. Increased Frequency of DRB 1 * 11: 01 in Anti - Hydroxymethylglutaryl-Coenzyme A Reductase - Associated Autoimmune Myopathy. Arthritis Care Res. 2012;64:1233-7.

14 Rothwell S, Cooper RG, Lundberg IE, et al. Immune-Array analysis in sporadic inclusion body myositis reveals HLA-DRB1 amino acid heterogeneity across the myositis spectrum. Arthritis Rheumatol 2017;69:1090-9.

15 Jia X, Han B, Onengut-Gumuscu S, et al. Imputing amino acid polymorphisms in human leukocyte antigens. PLoS One 2013:8:e64683.

16 Gregersen PK, Kosoy R, Lee AT, et al. Risk for myasthenia gravis maps to a ${ }^{151}$ Pro $\rightarrow$ Ala change in TNIP1 and to human leukocyte antigen-B*08. Ann Neurol 2012;72:927-35.

17 Betteridge ZE, Chinoy H, Cooper RG, et al. 175 myositis-specific autoantibodies rarely coexist with each other: an analysis of the Ukmyonet and Eumyonet cohorts. Rheumatology 2016;55

18 Chinoy H, Li CK-C, Platt H, et al. Genetic association study of NF-KB genes in UK Caucasian adult and juvenile onset idiopathic inflammatory myopathy. Rheumatology 2012:51:794-9.

19 Chinoy H, Salway F, John S, et al. Tumour necrosis factor-alpha single nucleotide polymorphisms are not independent of HLA class I in UK Caucasians with adult onset idiopathic inflammatory myopathies. Rheumatology 2007;46:1411-6.

20 Lintner KE, Patwardhan A, Rider LG, et al. Gene copy-number variations (CNVs) of complement $C 4$ and $C 4 A$ deficiency in genetic risk and pathogenesis of juvenile dermatomyositis. Ann Rheum Dis 2016:75:1599-606.

21 Menconi F, Osman R, Monti MC, et al. Shared molecular amino acid signature in the HLA-DR peptide binding pocket predisposes to both autoimmune diabetes and thyroiditis. Proceedings of the National Academy of Sciences 2010;107:16899-903.

22 Okada Y, Kim K, Han B, et al. Risk for ACPA-positive rheumatoid arthritis is driven by shared HLA amino acid polymorphisms in Asian and European populations. Hum $\mathrm{Mol}$ Genet 2014;23:6916-26.

23 Rider LG, Shamim E, Okada S, et al. Genetic risk and protective factors for idiopathic inflammatory myopathy in Koreans and American whites: a tale of two loci. Arthritis Rheum 1999;42:1285-90.

24 Trallero-Araguás E, Rodrigo-Pendás Jose Ángel, Selva-O'Callaghan A, et al. Usefulness of anti-p155 autoantibody for diagnosing cancer-associated dermatomyositis: a systematic review and meta-analysis. Arthritis Rheum 2012;64:523-32.

25 Fiorentino DF, Chung LS, Christopher-Stine L, et al. Most patients with cancerassociated dermatomyositis have antibodies to nuclear matrix protein NXP-2 or transcription intermediary factor $1 \gamma$. Arthritis Rheum 2013;65:2954-62.

26 Pinal-Fernandez I, Ferrer-Fabregas B, Trallero-Araguas E, et al. Tumour TIF1 mutations and loss of heterozygosity related to cancer-associated myositis. Rheumatology 2018;57:388-96.

27 Manlhiot C, Liang L, Tran D, et al. Assessment of an infectious disease history preceding juvenile dermatomyositis symptom onset. Rheumatology 2008;47:526-9.

28 Pachman LM, Lipton R, Ramsey-Goldman R, et al. History of infection before the onset of juvenile dermatomyositis: results from the National Institute of arthritis and musculoskeletal and skin diseases research registry. Arthritis Rheum 2005; $53: 166-72$

29 Shah M, Targoff IN, Rice MM, et al. Brief report: ultraviolet radiation exposure is associated with clinical and autoantibody phenotypes in juvenile myositis. Arthritis Rheum 2013;65:1934-41.

30 Miller FW, Lamb JA, Schmidt J, et al. Risk factors and disease mechanisms in myositis. Nat Rev Rheumatol 2018;14:255-68.

31 Betteridge Z, Chinoy H, New P. Validation of commercial myositis line blots. Global Conference on Myositis, Washington, 2017:14

32 Ghirardello A, Rampudda M, Ekholm L, et al. Diagnostic performance and validation of autoantibody testing in myositis by a commercial line blot assay. Rheumatology 2010:49:2370-4. 